\title{
Executive Summary: Standards of Medical Care in Diabetes-2013
}

\section{Current criteria for the diagnosis of diabetes}

- $\mathrm{AlC} \geq 6.5 \%$. The test should be performed in a laboratory using a method that is NGSP certified and standardized to the Diabetes Control and Complications Trial (DCCT) assay; or

- fasting plasma glucose (FPG) $\geq 126$ $\mathrm{mg} / \mathrm{dL}(7.0 \mathrm{mmol} / \mathrm{L})$. Fasting is defined as no caloric intake for at least $8 \mathrm{~h}$; or

- 2-h plasma glucose $\geq 200 \mathrm{mg} / \mathrm{dL}$ (11.1 $\mathrm{mmol} / \mathrm{L}$ ) during an oral glucose tolerance test (OGTT). The test should be performed as described by the World Health Organization, using a glucose load containing the equivalent of $75 \mathrm{~g}$ anhydrous glucose dissolved in water; or

- in a patient with classic symptoms of hyperglycemia or hyperglycemic crisis, a random plasma glucose $\geq 200 \mathrm{mg} / \mathrm{dL}$ (11.1 mmol/L);

- in the absence of unequivocal hyperglycemia, result should be confirmed by repeat testing.

\section{Testing for diabetes in asymptomatic patients}

- Testing to detect type 2 diabetes and prediabetes in asymptomatic people should be considered in adults of any age who are overweight or obese (BMI $\geq 25 \mathrm{~kg} / \mathrm{m}^{2}$ ) and who have one or more additional risk factors for diabetes (see Table 4 of the "Standards of Medical Care in Diabetes-2013"). In those without these risk factors, testing should begin at age 45 years. (B)

- If tests are normal, repeat testing at least at 3-year intervals is reasonable. (E)

- To test for diabetes or prediabetes, the AlC, FPG, or 75-g 2-h OGTT are appropriate. (B)

- In those identified with prediabetes, identify and, if appropriate, treat other cardiovascular disease (CVD) risk factors. (B)

\section{Screening for type 2 diabetes in children}

- Testing to detect type 2 diabetes and prediabetes should be considered in children and adolescents who are overweight and who have two or more additional risk factors for diabetes (see Table 5 of the "Standards of Medical Care in Diabetes-2013"). (E)

\section{Screening for type 1 diabetes}

- Consider referring relatives of those with type 1 diabetes for antibody testing for risk assessment in the setting of a clinical research study. (E)

\section{Detection and diagnosis of gestational diabetes mellitus}

- Screen for undiagnosed type 2 diabetes at the first prenatal visit in those with risk factors, using standard diagnostic criteria. (B)

- In pregnant women not previously known to have diabetes, screen for gestational diabetes mellitus (GDM) at 24-28 weeks of gestation, using a 75-g 2-h OGTT and the diagnostic cut points in Table 6 of the "Standards of Medical Care in Diabetes-2013." (B)

- Screen women with GDM for persistent diabetes at 6-12 weeks postpartum, using the OGTT and nonpregnancy diagnostic criteria. (E)

- Women with a history of GDM should have lifelong screening for the development of diabetes or prediabetes at least every 3 years. (B)

- Women with a history of GDM found to have prediabetes should receive lifestyle interventions or metformin to prevent diabetes. (A)

\section{Prevention/delay of type 2 diabetes}

- Patients with impaired glucose tolerance (IGT) (A), impaired fasting glucose cited, the use is educational and not for profit, and the work is not altered. See http://creativecommons.org/ licenses/by-nc-nd/3.0/ for details

(IFG) (E), or an AlC 5.7-6.4\% (E) should be referred to an effective ongoing support program targeting weight loss of $7 \%$ of body weight and increasing physical activity to at least 150 min/week of moderate activity such as walking.

- Follow-up counseling appears to be important for success. (B)

- Based on the cost-effectiveness of diabetes prevention, such programs should be covered by third-party payers. (B)

- Metformin therapy for prevention of type 2 diabetes may be considered in those with IGT (A), IFG (E), or an AlC $5.7-6.4 \%$ (E), especially for those with BMI $>35 \mathrm{~kg} / \mathrm{m}^{2}$, aged $<60$ years, and women with prior GDM. (A)

- At least annual monitoring for the development of diabetes in those with prediabetes is suggested. (E)

- Screening for and treatment of modifiable risk factors for CVD is suggested. (B)

\section{Glucose monitoring}

- Patients on multiple-dose insulin (MDI) or insulin pump therapy should do self-monitoring of blood glucose (SMBG) at least prior to meals and snacks, occasionally postprandially, at bedtime, prior to exercise, when they suspect low blood glucose, after treating low blood glucose until they are normoglycemic, and prior to critical tasks such as driving. (B)

- When prescribed as part of a broader educational context, SMBG results may be helpful to guide treatment decisions and/or patient self-management for patients using less frequent insulin injections or noninsulin therapies. (E)

- When prescribing SMBG, ensure that patients receive ongoing instruction and regular evaluation of SMBG technique and SMBG results, as well as their ability to use SMBG data to adjust therapy. (E)

- Continuous glucose monitoring (CGM) in conjunction with intensive insulin regimens can be a useful tool to lower AlC in selected adults (aged $\geq 25$ years) with type 1 diabetes. (A)

- Although the evidence for AlC lowering is less strong in children, teens, and 
younger adults, CGM may be helpful in these groups. Success correlates with adherence to ongoing use of the device. (C)

- CGM may be a supplemental tool to SMBG in those with hypoglycemia unawareness and/or frequent hypoglycemic episodes. (E)

\section{A1C}

- Perform the AlC test at least two times a year in patients who are meeting treatment goals (and who have stable glycemic control). (E)

- Perform the AlC test quarterly in patients whose therapy has changed or who are not meeting glycemic goals. (E)

- Use of point-of-care testing for AlC provides the opportunity for more timely treatment changes. (E)

\section{Clycemic goals in adults}

- Lowering A1C to below or around 7\% has been shown to reduce microvascular complications of diabetes, and if implemented soon after the diagnosis of diabetes is associated with long-term reduction in macrovascular disease. Therefore, a reasonable AlC goal for many nonpregnant adults is $<7 \%$. (B)

- Providers might reasonably suggest more stringent AlC goals (such as $<6.5 \%$ ) for selected individual patients, if this can be achieved without significant hypoglycemia or other adverse effects of treatment. Appropriate patients might include those with short duration of diabetes, long life expectancy, and no significant CVD. (C)

- Less stringent AlC goals (such as $<8 \%$ ) may be appropriate for patients with a history of severe hypoglycemia, limited life expectancy, advanced microvascular or macrovascular complications, extensive comorbid conditions, and those with long-standing diabetes in whom the general goal is difficult to attain despite diabetes self-management education (DSME), appropriate glucose monitoring, and effective doses of multiple glucose-lowering agents including insulin. (B)

\section{Pharmacological and overall approaches to treatment}

Insulin therapy for type 1 diabetes

- Most people with type 1 diabetes should be treated with MDI injections (three to four injections per day of basal and prandial insulin) or continuous subcutaneous insulin infusion (CSII). (A)
- Most people with type 1 diabetes should be educated in how to match prandial insulin dose to carbohydrate intake, premeal blood glucose, and anticipated activity. (E)

- Most people with type 1 diabetes should use insulin analogs to reduce hypoglycemia risk. (A)

- Consider screening those with type 1 diabetes for other autoimmune diseases (thyroid, vitamin B12 deficiency, celiac) as appropriate. (B)

\section{Pharmacological therapy for} hyperglycemia in type $\mathbf{2}$ diabetes

- Metformin, if not contraindicated and if tolerated, is the preferred initial pharmacological agent for type 2 diabetes. (A)

- In newly diagnosed type 2 diabetic patients with markedly symptomatic and/or elevated blood glucose levels or A1C, consider insulin therapy, with or without additional agents, from the outset. (E)

- If noninsulin monotherapy at maximal tolerated dose does not achieve or maintain the AlC target over 3-6 months, add a second oral agent, a glucagon-like peptide-1 (GLP-1) receptor agonist, or insulin. (A)

- A patient-centered approach should be used to guide choice of pharmacological agents. Considerations include efficacy, cost, potential side effects, effects on weight, comorbidities, hypoglycemia risk, and patient preferences. (E)

- Due to the progressive nature of type 2 diabetes, insulin therapy is eventually indicated for many patients with type 2 diabetes. (B)

\section{Medical nutrition therapy}

\section{General recommendations}

- Individuals who have prediabetes or diabetes should receive individualized medical nutrition therapy (MNT) as needed to achieve treatment goals, preferably provided by a registered dietitian familiar with the components of diabetes MNT. (A)

- Because MNT can result in cost-savings and improved outcomes (B), MNT should be adequately covered by insurance and other payers. (E)

\section{Energy balance, overweight, and obesity}

- Weight loss is recommended for all overweight or obese individuals who have or are at risk for diabetes. (A)
- For weight loss, either low-carbohydrate, low-fat calorie-restricted, or Mediterranean diets may be effective in the short term (up to 2 years). (A)

- For patients on low-carbohydrate diets, monitor lipid profiles, renal function, and protein intake (in those with nephropathy) and adjust hypoglycemic therapy as needed. (E)

- Physical activity and behavior modification are important components of weight loss programs and are most helpful in maintenance of weight loss. (B)

\section{Recommendations for primary prevention of type 2 diabetes}

- Among individuals at high risk for developing type 2 diabetes, structured programs that emphasize lifestyle changes that include moderate weight loss (7\% body weight) and regular physical activity (150 min/week), with dietary strategies including reduced calories and reduced intake of dietary fat, can reduce the risk for developing diabetes and are therefore recommended. (A)

- Individuals at risk for type 2 diabetes should be encouraged to achieve the U.S. Department of Agriculture (USDA) recommendation for dietary fiber ( $14 \mathrm{~g}$ fiber/1,000 kcal) and foods containing whole grains (one-half of grain intake). (B)

- Individuals at risk for type 2 diabetes should be encouraged to limit their intake of sugar-sweetened beverages (SSBs). (B)

\section{Recommendations for management of diabetes}

Macronutrients in diabetes management

- The mix of carbohydrate, protein, and fat may be adjusted to meet the metabolic goals and individual preferences of the person with diabetes. (C)

- Monitoring carbohydrate, whether by carbohydrate counting, choices, or experience-based estimation, remains a key strategy in achieving glycemic control. (B)

- Saturated fat intake should be $<7 \%$ of total calories. (B)

- Reducing intake of trans fat lowers LDL cholesterol and increases HDL cholesterol (A); therefore, intake of trans fat should be minimized. (E)

Other nutrition recommendations

- If adults with diabetes choose to use alcohol, they should limit intake to a moderate amount (one drink per day or less for adult women and two drinks per day or less for adult men) and 
should take extra precautions to prevent hypoglycemia. (E)

- Routine supplementation with antioxidants, such as vitamins $E$ and $C$ and carotene, is not advised because of lack of evidence of efficacy and concern related to long-term safety. (A)

- It is recommended that individualized meal planning include optimization of food choices to meet recommended dietary allowance (RDA)/dietary reference intake (DRI) for all micronutrients. (E)

\section{Diabetes self-management education and support}

- People with diabetes should receive DSME and diabetes self-management support (DSMS) according to National Standards for Diabetes Self-Management Education and Support when their diabetes is diagnosed and as needed thereafter. (B)

- Effective self-management and quality of life are the key outcomes of DSME and DSMS and should be measured and monitored as part of care. (C)

- DSME and DSMS should address psychosocial issues, since emotional well-being is associated with positive diabetes outcomes. (C)

- DSME and DSMS programs are appropriate venues for people with prediabetes to receive education and support to develop and maintain behaviors that can prevent or delay the onset of diabetes. (C)

- Because DSME and DSMS can result in cost-savings and improved outcomes (B), DSME and DSMS should be adequately reimbursed by third-party payers. (E)

\section{Physical activity}

- Adults with diabetes should be advised to perform at least $150 \mathrm{~min} /$ week of moderate-intensity aerobic physical activity (50-70\% of maximum heart rate), spread over at least 3 days/week with no more than 2 consecutive days without exercise. (A)

- In the absence of contraindications, adults with type 2 diabetes should be encouraged to perform resistance training at least twice per week. (A)

\section{Psychosocial assessment and cerre}

- It is reasonable to include assessment of the patient's psychological and social situation as an ongoing part of the medical management of diabetes. (E)
- Psychosocial screening and follow-up may include, but are not limited to, attitudes about the illness, expectations for medical management and outcomes, affect/mood, general and diabetes-related quality of life, resources (financial, social, and emotional), and psychiatric history. (E)

- Screen for psychosocial problems such as depression and diabetes-related distress, anxiety, eating disorders, and cognitive impairment when selfmanagement is poor. (B)

\section{Hypoglycemia}

- Individuals at risk for hypoglycemia should be asked about symptomatic and asymptomatic hypoglycemia at each encounter. (C)

- Glucose (15-20 g) is the preferred treatment for the conscious individual with hypoglycemia, although any form of carbohydrate that contains glucose may be used. If SMBG 15 min after treatment shows continued hypoglycemia, the treatment should be repeated. Once SMBG glucose returns to normal, the individual should consume a meal or snack to prevent recurrence of hypoglycemia. (E)

- Glucagon should be prescribed for all individuals at significant risk of severe hypoglycemia, and caregivers or family members of these individuals should be instructed on its administration. Glucagon administration is not limited to health care professionals. (E)

- Hypoglycemia unawareness or one or more episodes of severe hypoglycemia should trigger re-evaluation of the treatment regimen. (E)

- Insulin-treated patients with hypoglycemia unawareness or an episode of severe hypoglycemia should be advised to raise their glycemic targets to strictly avoid further hypoglycemia for at least several weeks, to partially reverse hypoglycemia unawareness, and to reduce risk of future episodes. (A)

- Ongoing assessment of cognitive function is suggested with increased vigilance for hypoglycemia by the clinician, patient, and caregivers if low cognition and/or declining cognition is found. (B)

\section{Bariatric surgery}

- Bariatric surgery may be considered for adults with $\mathrm{BMI} \geq 35 \mathrm{~kg} / \mathrm{m}^{2}$ and type 2 diabetes, especially if the diabetes or associated comorbidities are difficult to control with lifestyle and pharmacological therapy. (B)
- Patients with type 2 diabetes who have undergone bariatric surgery need lifelong lifestyle support and medical monitoring. (B)

- Although small trials have shown glycemic benefit of bariatric surgery in patients with type 2 diabetes and BMI $30-35 \mathrm{~kg} / \mathrm{m}^{2}$, there is currently insufficient evidence to generally recommend surgery in patients with BMI $<35$ $\mathrm{kg} / \mathrm{m}^{2}$ outside of a research protocol. (E)

- The long-term benefits, cost-effectiveness, and risks of bariatric surgery in individuals with type 2 diabetes should be studied in well-designed controlled trials with optimal medical and lifestyle therapy as the comparator. (E)

\section{Immunization}

- Annually provide an influenza vaccine to all diabetic patients $\geq 6$ months of age. (C)

- Administer pneumococcal polysaccharide vaccine to all diabetic patients $\geq 2$ years of age. A one-time revaccination is recommended for individuals $>64$ years of age previously immunized when they were $<65$ years of age if the vaccine was administered $>5$ years ago. Other indications for repeat vaccination include nephrotic syndrome, chronic renal disease, and other immunocompromised states, such as after transplantation. (C)

- Administer hepatitis B vaccination to unvaccinated adults with diabetes who are aged 19 through 59 years. (C)

- Consider administering hepatitis B vaccination to unvaccinated adults with diabetes who are aged $\geq 60$ years. (C)

\section{Hypertension/blood pressure control}

\section{Screening and diagnosis}

- Blood pressure should be measured at every routine visit. Patients found to have elevated blood pressure should have blood pressure confirmed on a separate day. (B)

\section{Goals}

- People with diabetes and hypertension should be treated to a systolic blood pressure goal of $<140 \mathrm{mmHg}$. (B)

- Lower systolic targets, such as <130 $\mathrm{mmHg}$, may be appropriate for certain individuals, such as younger patients, if it can be achieved without undue treatment burden. (C)

- Patients with diabetes should be treated to a diastolic blood pressure $<80$ mmHg. (B) 


\section{Treatment}

- Patients with a blood pressure $>120 / 80$ mmHg should be advised on lifestyle changes to reduce blood pressure. (B)

- Patients with confirmed blood pressure $\geq 140 / 80 \mathrm{mmHg}$ should, in addition to lifestyle therapy, have prompt initiation and timely subsequent titration of pharmacological therapy to achieve blood pressure goals. (B)

- Lifestyle therapy for elevated blood pressure consists of weight loss, if overweight; Dietary Approaches to Stop Hypertension (DASH)-style dietary pattern including reducing sodium and increasing potassium intake; moderation of alcohol intake; and increased physical activity. (B)

- Pharmacological therapy for patients with diabetes and hypertension should be with a regimen that includes either an ACE inhibitor or an angiotensin receptor blocker (ARB). If one class is not tolerated, the other should be substituted. (C)

- Multiple-drug therapy (two or more agents at maximal doses) is generally required to achieve blood pressure targets. (B)

- Administer one or more antihypertensive medications at bedtime. (A)

- If ACE inhibitors, ARBs, or diuretics are used, serum creatinine/estimated glomerular filtration rate (eGFR) and serum potassium levels should be monitored. (E)

- In pregnant patients with diabetes and chronic hypertension, blood pressure target goals of 110-129/65-79 mmHg are suggested in the interest of longterm maternal health and minimizing impaired fetal growth. ACE inhibitors and ARBs are contraindicated during pregnancy. (E)

\section{Dyslipidemia/lipid manciement}

\section{Screening}

- In most adult patients with diabetes, measure fasting lipid profile at least annually. (B)

- In adults with low-risk lipid values (LDL cholesterol <100 mg/dL, HDL cholesterol $>50 \mathrm{mg} / \mathrm{dL}$, and triglycerides $<150 \mathrm{mg} / \mathrm{dL}$ ), lipid assessments may be repeated every 2 years. (E)

\section{Treatment recommendations and goals}

- Lifestyle modification focusing on the reduction of saturated fat, trans fat, and cholesterol intake; increase of n-3 fatty acids, viscous fiber and plant stanols/ sterols; weight loss (if indicated); and increased physical activity should be recommended to improve the lipid profile in patients with diabetes. (A)

- Statin therapy should be added to lifestyle therapy, regardless of baseline lipid levels, for diabetic patients:

- with overt CVD (A)

- without CVD who are over the age of 40 years and have one or more other CVD risk factors (family history of CVD, hypertension, smoking, dyslipidemia, or albuminuria). (A)

- For lower-risk patients than the above (e.g., without overt CVD and under the age of 40 years), statin therapy should be considered in addition to lifestyle therapy if LDL cholesterol remains above $100 \mathrm{mg} / \mathrm{dL}$ or in those with multiple CVD risk factors. (C)

- In individuals without overt CVD, the goal is LDL cholesterol $<100 \mathrm{mg} / \mathrm{dL}$ ( $2.6 \mathrm{mmol} / \mathrm{L}$ ). (B)

- In individuals with overt CVD, a lower LDL cholesterol goal of $<70 \mathrm{mg} / \mathrm{dL}$ $(1.8 \mathrm{mmol} / \mathrm{L})$, using a high dose of a statin, is an option. (B)

- If drug-treated patients do not reach the above targets on maximal tolerated statin therapy, a reduction in LDL cholesterol of $\sim 30-40 \%$ from baseline is an alternative therapeutic goal. (B)

- Triglyceride levels $<150 \mathrm{mg} / \mathrm{dL}$ (1.7 $\mathrm{mmol} / \mathrm{L}$ ) and HDL cholesterol $>40$ $\mathrm{mg} / \mathrm{dL}(1.0 \mathrm{mmol} / \mathrm{L})$ in men and $>50$ $\mathrm{mg} / \mathrm{dL}(1.3 \mathrm{mmol} / \mathrm{L})$ in women are desirable (C). However, LDL cholesterol-targeted statin therapy remains the preferred strategy. (A)

- Combination therapy has been shown not to provide additional cardiovascular benefit above statin therapy alone and is not generally recommended. (A)

- Statin therapy is contraindicated in pregnancy. (B)

\section{Antiplatelet agents}

- Consider aspirin therapy (75-162 mg/ day) as a primary prevention strategy in those with type 1 or type 2 diabetes at increased cardiovascular risk (10-year risk $>10 \%$ ). This includes most men aged $>50$ years or women aged $>60$ years who have at least one additional major risk factor (family history of CVD, hypertension, smoking, dyslipidemia, or albuminuria). (C)

- Aspirin should not be recommended for CVD prevention for adults with diabetes at low CVD risk (10-year CVD risk $<5 \%$, such as in men aged $<50$ years and women aged $<60$ years with no major additional CVD risk factors), since the potential adverse effects from bleeding likely offset the potential benefits. (C)

- In patients in these age-groups with multiple other risk factors (e.g., 10year risk $5-10 \%$ ), clinical judgment is required. (E)

- Use aspirin therapy (75-162 mg/day) as a secondary prevention strategy in those with diabetes with a history of CVD. (A)

- For patients with CVD and documented aspirin allergy, clopidogrel (75 mg/day) should be used. (B)

- Combination therapy with aspirin (75-162 mg/day) and clopidogrel (75 $\mathrm{mg} /$ day) is reasonable for up to a year after an acute coronary syndrome. (B)

\section{Smoking cessation}

- Advise all patients not to smoke or use tobacco products. (A)

- Include smoking cessation counseling and other forms of treatment as a routine component of diabetes care. (B)

\section{Coronary heart disease screening and treatment}

\section{Screening}

- In asymptomatic patients, routine screening for coronary artery disease (CAD) is not recommended, as it does not improve outcomes as long as CVD risk factors are treated. (A)

\section{Treatment}

- In patients with known CVD, consider ACE inhibitor therapy (C) and use aspirin and statin therapy (A) (if not contraindicated) to reduce the risk of cardiovascular events. In patients with a prior myocardial infarction, $\beta$-blockers should be continued for at least 2 years after the event. (B)

- Avoid thiazolidinedione treatment in patients with symptomatic heart failure. (C)

- Metformin may be used in patients with stable congestive heart failure (CHF) if renal function is normal. It should be avoided in unstable or hospitalized patients with $\mathrm{CHF}$. (C)

\section{Nephropathy screening and treatment}

General recommendations

- To reduce the risk or slow the progression of nephropathy, optimize glucose control. (A) 
- To reduce the risk or slow the progression of nephropathy, optimize blood pressure control. (A)

\section{Screening}

- Perform an annual test to assess urine albumin excretion in type 1 diabetic patients with diabetes duration of $\geq 5$ years and in all type 2 diabetic patients starting at diagnosis. (B)

- Measure serum creatinine at least annually in all adults with diabetes regardless of the degree of urine albumin excretion. The serum creatinine should be used to estimate glomerular filtration rate (GFR) and stage the level of chronic kidney disease (CKD), if present. (E)

\section{Treatment}

- In the treatment of the nonpregnant patient with modestly elevated (30$299 \mathrm{mg} /$ day) (C) or higher levels ( $\geq 300 \mathrm{mg} /$ day) of urinary albumin excretion (A), either ACE inhibitors or ARBs are recommended.

- Reduction of protein intake to 0.8-1.0 $\mathrm{g} / \mathrm{kg}$ body wt per day in individuals with diabetes and the earlier stages of CKD and to $0.8 \mathrm{~g} / \mathrm{kg}$ body wt per day in the later stages of CKD may improve measures of renal function (urine albumin excretion rate, GFR) and is recommended. (C)

- When ACE inhibitors, ARBs, or diuretics are used, monitor serum creatinine and potassium levels for the development of increased creatinine or changes in potassium. (E)

- Continued monitoring of urine albumin excretion to assess both response to therapy and progression of disease is reasonable. (E)

- When eGFR is $<60 \mathrm{~mL} / \mathrm{min} / 1.73 \mathrm{~m}^{2}$, evaluate and manage potential complications of CKD. (E)

- Consider referral to a physician experienced in the care of kidney disease for uncertainty about the etiology of kidney disease, difficult management issues, or advanced kidney disease. (B)

\section{Retinopathy screening and treatment}

\section{General recommendations}

- To reduce the risk or slow the progression of retinopathy, optimize glycemic control. (A)

- To reduce the risk or slow the progression of retinopathy, optimize blood pressure control. (A)

\section{Screening}

- Adults and children aged $\geq 10$ years with type 1 diabetes should have an initial dilated and comprehensive eye examination by an ophthalmologist or optometrist within 5 years after the onset of diabetes. (B)

- Patients with type 2 diabetes should have an initial dilated and comprehensive eye examination by an ophthalmologist or optometrist shortly after the diagnosis of diabetes. (B)

- Subsequent examinations for type 1 and type 2 diabetic patients should be repeated annually by an ophthalmologist or optometrist. Less frequent exams (every 2-3 years) may be considered following one or more normal eye exams. Examinations will be required more frequently if retinopathy is progressing. (B)

- High-quality fundus photographs can detect most clinically significant diabetic retinopathy. Interpretation of the images should be performed by a trained eye care provider. While retinal photography may serve as a screening tool for retinopathy, it is not a substitute for a comprehensive eye exam, which should be performed at least initially and at intervals thereafter as recommended by an eye care professional. (E)

- Women with pre-existing diabetes who are planning pregnancy or who have become pregnant should have a comprehensive eye examination and be counseled on the risk of development and/or progression of diabetic retinopathy. Eye examination should occur in the first trimester with close follow-up throughout pregnancy and for 1 year postpartum. (B)

\section{Treatment}

- Promptly refer patients with any level of macular edema, severe nonproliferative diabetic retinopathy (NPDR), or any proliferative diabetic retinopathy (PDR) to an ophthalmologist who is knowledgeable and experienced in the management and treatment of diabetic retinopathy. (A)

- Laser photocoagulation therapy is indicated to reduce the risk of vision loss in patients with high-risk PDR, clinically significant macular edema, and in some cases of severe NPDR. (A)

- Anti-vascular endothelial growth factor (VEGF) therapy is indicated for diabetic macular edema. (A)
- The presence of retinopathy is not a contraindication to aspirin therapy for cardioprotection, as this therapy does not increase the risk of retinal hemorrhage. (A)

\section{Neuropathy screening and treatment}

- All patients should be screened for distal symmetric polyneuropathy (DPN) starting at diagnosis of type 2 diabetes and 5 years after the diagnosis of type 1 diabetes and at least annually thereafter, using simple clinical tests. (B)

- Electrophysiological testing is rarely needed, except in situations where the clinical features are atypical. (E)

- Screening for signs and symptoms of cardiovascular autonomic neuropathy (CAN) should be instituted at diagnosis of type 2 diabetes and 5 years after the diagnosis of type 1 diabetes. Special testing is rarely needed and may not affect management or outcomes. (E)

- Medications for the relief of specific symptoms related to painful DPN and autonomic neuropathy are recommended, as they improve the quality of life of the patient. (E)

\section{Foot care}

- For all patients with diabetes, perform an annual comprehensive foot examination to identify risk factors predictive of ulcers and amputations. The foot examination should include inspection, assessment of foot pulses, and testing for loss of protective sensation (LOPS) (10-g monofilament plus testing any one of the following: vibration using $128-\mathrm{Hz}$ tuning fork, pinprick sensation, ankle reflexes, or vibration perception threshold). (B)

- Provide general foot self-care education to all patients with diabetes. (B)

- A multidisciplinary approach is recommended for individuals with foot ulcers and high-risk feet, especially those with a history of prior ulcer or amputation. (B)

- Refer patients who smoke, have LOPS and structural abnormalities, or have a history of prior lower-extremity complications to foot care specialists for ongoing preventive care and lifelong surveillance. (C)

- Initial screening for peripheral arterial disease (PAD) should include a history for claudication and an assessment of the pedal pulses. Consider obtaining an ankle-brachial index (ABI), as many patients with PAD are asymptomatic. (C) 
- Refer patients with significant claudication or a positive ABI for further vascular assessment and consider exercise, medications, and surgical options. (C)

\section{Assessment of common comorbid conditions}

- For patients with risk factors, signs, or symptoms, consider assessment and treatment for common diabetesassociated conditions (see Table 14 of the "Standards of Medical Care in Diabetes-2013"). (B)

\section{Children and adolescents}

- As is the case for all children, children with diabetes or prediabetes should be encouraged to engage in at least $60 \mathrm{~min}$ of physical activity each day. (B)

\section{Type 1 diabetes}

\section{Glycemic control}

- Consider age when setting glycemic goals in children and adolescents with type 1 diabetes. (E)

\section{Screening and management of chronic complications in children and adolescents with type 1 diabetes}

Nephropathy

- Annual screening for microalbuminuria, with a random spot urine sample for albumin-to-creatinine ratio (ACR), should be considered once the child is 10 years of age and has had diabetes for 5 years. (B)

- Treatment with an ACE inhibitor, titrated to normalization of albumin excretion, should be considered when elevated ACR is subsequently confirmed on two additional specimens from different days. (E)

\section{Hypertension}

- Blood pressure should be measured at each routine visit. Children found to have high-normal blood pressure or hypertension should have blood pressure confirmed on a separate day. (B)

- Initial treatment of high-normal blood pressure (systolic or diastolic blood pressure consistently above the 90th percentile for age, sex, and height) includes dietary intervention and exercise, aimed at weight control and increased physical activity, if appropriate. If target blood pressure is not reached with 3-6 months of lifestyle intervention, pharmacological treatment should be considered. (E)
- Pharmacological treatment of hypertension (systolic or diastolic blood pressure consistently above the 95th percentile for age, sex, and height or consistently $>130 / 80 \mathrm{mmHg}$, if $95 \%$ exceeds that value) should be considered as soon as the diagnosis is confirmed. (E)

- ACE inhibitors should be considered for the initial treatment of hypertension, following appropriate reproductive counseling due to its potential teratogenic effects. (E)

- The goal of treatment is a blood pressure consistently $<130 / 80$ or below the 90th percentile for age, sex, and height, whichever is lower. (E)

\section{Dyslipidemia}

\section{Screening}

- If there is a family history of hypercholesterolemia or a cardiovascular event before age 55 years, or if family history is unknown, then consider obtaining a fasting lipid profile on children $>2$ years of age soon after diagnosis (after glucose control has been established). If family history is not of concern, then consider the first lipid screening at puberty ( $\geq 10$ years of age). For children diagnosed with diabetes at or after puberty, consider obtaining a fasting lipid profile soon after the diagnosis (after glucose control has been established). (E)

- For both age-groups, if lipids are abnormal, annual monitoring is reasonable. If LDL cholesterol values are within the accepted risk levels $(<100 \mathrm{mg} / \mathrm{dL}$ [2.6 mmol/L]), a lipid profile repeated every 5 years is reasonable. (E)

Treatment

- Initial therapy may consist of optimization of glucose control and MNT using a Step 2 American Heart Association (AHA) diet aimed at a decrease in the amount of saturated fat in the diet. (E)

- After the age of 10 years, the addition of a statin in patients who, after MNT and lifestyle changes, have LDL cholesterol $>160 \mathrm{mg} / \mathrm{dL}$ (4.1 mmol/L) or LDL cholesterol $>130 \mathrm{mg} / \mathrm{dL} \quad(3.4$ $\mathrm{mmol} / \mathrm{L}$ ) and one or more CVD risk factors is reasonable. (E)

- The goal of therapy is an LDL cholesterol value $<100 \mathrm{mg} / \mathrm{dL}(2.6 \mathrm{mmol} / \mathrm{L})$. (E)

\section{Retinopathy}

- The first ophthalmologic examination should be obtained once the child is $\geq 10$ years of age and has had diabetes for 3-5 years. (B)

- After the initial examination, annual routine follow-up is generally recommended.
Less frequent examinations may be acceptable on the advice of an eye care professional. (E)

\section{Celiac disease}

- Consider screening children with type 1 diabetes for celiac disease by measuring tissue transglutaminase or antiendomysial antibodies, with documentation of normal total serum IgA levels, soon after the diagnosis of diabetes. (E)

- Testing should be considered in children with growth failure, failure to gain weight, weight loss, diarrhea, flatulence, abdominal pain, or signs of malabsorption or in children with frequent unexplained hypoglycemia or deterioration in glycemic control. (E)

- Consider referral to a gastroenterologist for evaluation with possible endoscopy and biopsy for confirmation of celiac disease in asymptomatic children with positive antibodies. (E)

- Children with biopsy-confirmed celiac disease should be placed on a gluten-free diet and have consultation with a dietitian experienced in managing both diabetes and celiac disease. (B)

\section{Hypothyroidism}

- Consider screening children with type 1 diabetes for thyroid peroxidase and thyroglobulin antibodies soon after diagnosis. (E)

- Measuring thyroid-stimulating hormone (TSH) concentrations soon after diagnosis of type 1 diabetes, after metabolic control has been established, is reasonable. If normal, consider rechecking every 1-2 years, especially if the patient develops symptoms of thyroid dysfunction, thyromegaly, or an abnormal growth rate. (E)

\section{Transition from pediatric to adult care}

- As teens transition into emerging adulthood, health care providers and families must recognize their many vulnerabilities (B) and prepare the developing teen, beginning in early to mid adolescence and at least 1 year prior to the transition. (E)

- Both pediatricians and adult health care providers should assist in providing support and links to resources for the teen and emerging adult. (B)

\section{Preconception care}

- AlC levels should be as close to normal as possible $(<7 \%)$ in an individual patient before conception is attempted. (B) 
- Starting at puberty, preconception counseling should be incorporated in the routine diabetes clinic visit for all women of childbearing potential. (C)

- Women with diabetes who are contemplating pregnancy should be evaluated and, if indicated, treated for diabetic retinopathy, nephropathy, neuropathy, and CVD. (B)

- Medications used by such women should be evaluated prior to conception, since drugs commonly used to treat diabetes and its complications may be contraindicated or not recommended in pregnancy, including statins, ACE inhibitors, ARBs, and most noninsulin therapies. (E)

- Since many pregnancies are unplanned, consider the potential risks and benefits of medications that are contraindicated in pregnancy in all women of childbearing potential and counsel women using such medications accordingly. (E)

\section{Older adults}

- Older adults who are functional, cognitively intact, and have significant life expectancy should receive diabetes care with goals similar to those developed for younger adults. (E)

- Glycemic goals for some older adults might reasonably be relaxed, using individual criteria, but hyperglycemia leading to symptoms or risk of acute hyperglycemic complications should be avoided in all patients. (E)

- Other cardiovascular risk factors should be treated in older adults with consideration of the time frame of benefit and the individual patient. Treatment of hypertension is indicated in virtually all older adults, and lipid and aspirin therapy may benefit those with life expectancy at least equal to the time frame of primary or secondary prevention trials. (E)

- Screening for diabetes complications should be individualized in older adults, but particular attention should be paid to complications that would lead to functional impairment. (E)

\section{Cystic fibrosis-related diabetes}

- Annual screening for cystic fibrosisrelated diabetes (CFRD) with OGTT should begin by age 10 years in all patients with cystic fibrosis who do not have CFRD (B). Use of AlC as a screening test for CFRD is not recommended. (B)

- During a period of stable health, the diagnosis of CFRD can be made in cystic fibrosis patients according to usual glucose criteria. (E)

- Patients with CFRD should be treated with insulin to attain individualized glycemic goals. (A)

- Annual monitoring for complications of diabetes is recommended, beginning 5 years after the diagnosis of CFRD. (E)

\section{Diabetes care in the hospital}

- All patients with diabetes admitted to the hospital should have their diabetes clearly identified in the medical record. (E)

- All patients with diabetes should have an order for blood glucose monitoring, with results available to all members of the health care team. (E)

- Goals for blood glucose levels:

- Critically ill patients: Insulin therapy should be initiated for treatment of persistent hyperglycemia starting at a threshold of no greater than $180 \mathrm{mg} / \mathrm{dL}(10 \mathrm{mmol} / \mathrm{L})$. Once insulin therapy is started, a glucose range of $140-180 \mathrm{mg} / \mathrm{dL}$ (7.8-10 $\mathrm{mmol} / \mathrm{L})$ is recommended for the majority of critically ill patients. (A)

- More stringent goals, such as 110$140 \mathrm{mg} / \mathrm{dL}$ (6.1-7.8 mmol/L) may be appropriate for selected patients, as long as this can be achieved without significant hypoglycemia. (C)

- Critically ill patients require an intravenous insulin protocol that has demonstrated efficacy and safety in achieving the desired glucose range without increasing risk for severe hypoglycemia. (E)

- Non-critically ill patients: There is no clear evidence for specific blood glucose goals. If treated with insulin, the premeal blood glucose targets generally $<140 \mathrm{mg} / \mathrm{dL}$ (7.8 $\mathrm{mmol} / \mathrm{L})$ with random blood glucose $<180 \mathrm{mg} / \mathrm{dL}(10.0 \mathrm{mmol} / \mathrm{L})$ are reasonable, provided these targets can be safely achieved. More stringent targets may be appropriate in stable patients with previous tight glycemic control. Less stringent targets may be appropriate in those with severe comorbidities. (E)
- Scheduled subcutaneous insulin with basal, nutritional, and correction components is the preferred method for achieving and maintaining glucose control in non-critically ill patients. (C)

- Glucose monitoring should be initiated in any patient not known to be diabetic who receives therapy associated with high risk for hyperglycemia, including high-dose glucocorticoid therapy, initiation of enteral or parenteral nutrition, or other medications such as octreotide or immunosuppressive medications (B). If hyperglycemia is documented and persistent, consider treating such patients to the same glycemic goals as patients with known diabetes. (E)

- A hypoglycemia management protocol should be adopted and implemented by each hospital or hospital system. A plan for preventing and treating hypoglycemia should be established for each patient. Episodes of hypoglycemia in the hospital should be documented in the medical record and tracked. (E)

- Consider obtaining an A1C on patients with diabetes admitted to the hospital if the result of testing in the previous 2-3 months is not available. (E)

- Consider obtaining an AlC in patients with risk factors for undiagnosed diabetes who exhibit hyperglycemia in the hospital. (E)

- Patients with hyperglycemia in the hospital who do not have a prior diagnosis of diabetes should have appropriate plans for follow-up testing and care documented at discharge. (E)

\section{Strategies for improving diabetes care}

- Care should be aligned with components of the Chronic Care Model (CCM) to ensure productive interactions between a prepared proactive practice team and an informed activated patient. (A)

- When feasible, care systems should support team-based care, community involvement, patient registries, and embedded decision support tools to meet patient needs. (B)

- Treatment decisions should be timely and based on evidence-based guidelines that are tailored to individual patient preferences, prognoses, and comorbidities. (B)

- A patient-centered communication style should be employed that incorporates patient preferences, assesses literacy and numeracy, and addresses cultural barriers to care. (B) 